\section{Entrevista al Dr. William Giannobile, editor jefe de la Journal of Dental Research}

\section{Interview Dr. William Giannobile Editor-in- Chief Journal of Dental Research}

\section{Resumen}

Para el Dr. William Giannobile, experto reconocido internacionalmente en el campo de la ingeniería de tejidos dentales, el servir como editor jefe de la Journal of Dental Research $(J D R)$ ha sido una oportunidad increíble y uno de los esfuerzos más gratificantes de su carrera profesional. Ha cumplido los objetivos principales que se le propusieron como editor de esta revista y se siente privilegiado de haber podido trabajar en conjunto con editores muy capaces y con una visión de futuro increíble que han hecho de la $J D R$ la principal fuente de difusión en investigación durante más de un siglo. Bajo el liderazgo del Dr. Giannobile, la JDR se ha esforzado por alcanzar la calidad de las mejores revistas de investigación en Medicina y Ciencias Biológicas publicando los resultados de diferentes investigaciones, pero también estimulando el espíritu de investigación y promoviendo el desarrollo de investigación original en Estomatología.

Palabras clave: Entrevista; Ingeniería de tejidos; Periodoncia (fuente: DeCS BIREME).

\section{Entrevista}

\author{
Maria Eugenia Guerrero Acevedo 1,a \\ 1 Universidad Nacional Mayor de San Marcos, \\ Facultad de Odontología, Departamento \\ Académico Médico Quirúrgico. Lima, Perú. \\ a PhD en Biomedical Science.

\section{Correspondencia:} \\ Maria Eugenia Guerrero Acevedo \\ Correo electrónico: mguerreroac@unmsm.edu.pe \\ Universidad Nacional Mayor de San Marcos \\ Facultad de Odontología. Calle Germán \\ Amézaga 375. Lima 1, Perú.
}

Conflicto de intereses: la autora declara no tener conflictos de interés.

Fuente de financiamiento: autofinanciado.

Recibido: 15/10/19

Aceptado: 21/10/19

Publicado: 27/11/19

\begin{abstract}
For the Dr. William Giannobile, an internationally-recognized expert in the field of dental tissue engineering, serving as JDR Editor-in-Chief has been an amazing opportunity and one of the most rewarding endeavors in his professional career. He feel privileged to have been able to follow such capable and forward-thinking editors who have made the JDR the leading source of research findings for over a century. Under Dr. Giannobile's leadership, the JDR has strived to match the quality of the best research journals in medicine and life sciences by publishing the results of different investigations, but also stimulating the spirit of research; promoting the development of original research in Stomatology.
\end{abstract}

Keywords: Interview; Tissue engineering; Periodontics (source: MeSH NLM).

( ) Los autores. Este artículo es publicado por la revista Odontología Sanmarquina de la Facultad de Odontología, Universidad Nacional Mayor de San Marcos. Este es un artículo de acceso abierto, distribuido bajo los términos de la licencia Creative Commons Atribucion - No Comercia_Compartir Igual 4.0 Internacional. (http://creativecommons.org/licenses/by-nc-sa/4.0/) que permite el uso no comercial, distribución y reproducción en cualquier medio, siempre que la obra original sea debidamente citada. 


\section{Introducción}

William Giannobile recibió el premio William K. y Mary Anne Najjar de Odontología e Ingeniería Biomédica en la Universidad de Michigan. Es jefe del Departamento de Periodoncia y Medicina Oral de la Facultad de Odontología de la Universidad de Michigan. El Dr. Giannobile es un experto reconocido internacionalmente en el campo de la ingeniería de tejidos dentales. También es autor de libros y numerosos artículos relevantes para el campo clínico y de investigación en salud oral, desempeńándose actualmente como editor de la Journal of Dental Research $(J D R)$ desde el 2010.

\section{Entrevista}

Profesor Giannobile Ud. tiene varias publicaciones cientificas en el área de Periodoncia que son de gran ayuda a la comunidad odontológica. ¿Cuál es la línea de investigación que a Ud. más le apasiona? ¿Está trabajando en una nueva publicación?

Me llena de alegría poder dar esta entrevista a su grupo. La línea de investigación en la que he trabajado durante los últimos años es en el campo de la Medicina Regenerativa. En esta área, identificamos nuevas tendencias para regenerar hueso alrededor de los implantes dentales. Una de nuestras investigaciones más recientes consiste en utilizar diferentes anticuerpos neutralizadores de la molécula llamada: esclerostina, una proteína secretada por los osteocitos que limita la formación excesiva de hueso, inhibiendo en parte la actividad osteoblástica. Hemos estudiado recientemente la molécula llamada romosozumab que es un anticuerpo monoclonal anti-esclerostina. Hemos trabajado en la misma molécula desde hace casi 10 ańos para tratar la enfermedad periodontal y regenerar hueso alrededor de los implantes dentales, actualmente continuamos haciendo estudios al respecto.

¿Cuántos libros publicados tiene, y cuál es el que le brindó más satisfacciones profesionales?

He recibido la invitación para participar en diferentes proyectos para elaborar libros. Probablemente uno de los que me dio más satisfacciones fue uno de los primeros, titulado "Clinical Research in Oral Health". Escribí este libro con Robert J. Genco y Brian Burt y fue publicado en el 2010, fue uno de los primeros libros en desarrollar la metodología a seguir en investigación clínica y tuve la oportunidad de ser editor de este libro. A través de los años, he sido editor y co-editor de ocho libros. En la actualidad estoy trabajando en el libro: Clinical Periodontology and Implant Dentistry. Los editores son Jan Lindhe y Niklaus P. Lang, tiene dos tomos y estoy trabajando un capítulo en colaboración con Niklaus P. Lang, Tord Berglund y Mariano Sanz.

¿Cuál es su mayor objetivo profesional? ¿Qué es lo que le da más satisfacción de su trabajo como editor jefe de la JDR?

Mi mayor objetivo es poder ayudar a las personas a sacar lo mejor de sí mismas, doy soporte a mis estudiantes para la realización de sus logros personales. Por otro lado, en mi rol de editor de la $J D R$, me interesa dar el soporte necesario para poder difundir nuevos conocimientos.

¿Cómo llegó a ser editor jefe de la JDR? ¿Qué lo motivó a
asumir esta responsabilidad tan importante?

Gracias por realizar esa pregunta; a través de los años he estado inmerso en distintas fases del proceso editorial. Hace algunos años integré el Editorial Board de la Journal of Periodontology. En ese período, fui conociendo más sobre el proceso de revisión y descubrí que me gustaba realizar el rol de revisor y dar las sugerencias para la mejora de las publicaciones. Luego, algunos años después fui afortunado ya que Robert J. Genco me nombró editor asociado de la Journal of Periodontology, es allí donde tuve la oportunidad de conocer más sobre las decisiones de aceptación o rechazo de los artículos publicados, por casi 10 años. Luego, fui editor asociado de la biblioteca abierta de ciencias, PLOS ONE. Después de algún tiempo apliqué a la posición de editor de la $J D R$, este fue un proceso muy competitivo y agradezco el soporte del Directorio de la International Academy of Dental Research. Durante estos últimos 10 años he dado lo mejor de mí y espero haber contribuido en el avance de la revista durante todo este tiempo.

¿Por qué es tan difícil publicar en la JDR, cuál es la proporción de artículos rechazados?

En los últimos años, la revista se ha tornado cada vez más competitiva. Uno de los retos y alegrías que tenemos es que la revista publica tópicos sobre las diferentes especialidades cráneo-faciales. En la actualidad, recibimos alrededor de 1200 manuscritos por ańo. Nuestra tasa de aceptación es del 15\% de los artículos recibidos. También tenemos revisiones invitadas y éstas tienen una tasa de aceptación más alta.

¿Qué recomendaciones daría a la para la comunidad odontológica latinoamericana que desea ver sus trabajos publicados en la JDR?

La comunidad en Latinoamérica se ha ido expandiendo en términos de investigación y dentro del área odontológica. A través de los años han surgido grandes líderes en Latinoamérica. Uno de ellos es nuestro reciente editor asociado, Gustavo Garlet, de la ciudad de Sao Paulo, que ha contribuido mucho en nuestra revista. También, creo que con el aumento de los miembros de la International Association for Dental Research (IADR) provenientes de diferentes regiones, vemos cada vez más contribuciones de alrededor del mundo. En nuestra revista, creo que las personas que son más exitosas en las diferentes regiones han aprovechado el hecho de contar con grandes poblaciones específicas de pacientes, condición que puede extrapolarse a otras regiones del planeta con investigación de alto impacto en el área de epidemiología, patología y mecanismos biológicos. Si observan las últimas contribuciones que hemos tenido, Latinoamérica ha expandido sus áreas de publicación hacia las áreas de ciencias biológicas y clínicas. Ciertamente las alianzas colaborativas han sido variables y han contribuido en este sentido. Vemos que es cada vez más frecuente que investigadores de todo el planeta publiquen en colabora- 
ción con investigadores latinoamericanos dando origen a publicaciones importantes.

¿Cuál es el procedimiento cuando se retracta una publicación cientifica en su revista?

Este es un tópico muy sensible y creo que como editor de una revista tenemos que ser muy cuidadosos y asegurarnos de estar publicando las mejores investigaciones. Reconocemos también, que hay una gran variedad de razones por las que tenemos que retractar una publicación. En muchas ocasiones, se puede realizar un corrigendum o erratum, cuando se ha dejado fuera información importante. Algunas veces esto puede ser clarificado en una carta al editor. En el caso de retractar una publicación, generalmente se da cuando se detecta una mala conducta ética como fabricación de datos o publicaciones redundantes, que es cuando hay artículos idénticos. Afortunadamente, estoy absolutamente agradecido a los excelentes revisores que hemos tenido en los últimos años, quienes se dan el tiempo de realizar comentarios sobre los trabajos que reciben antes de aceptar los manuscritos. Sin embargo, en algunos casos hemos tenido que retractar algunas publicaciones debido al hallazgo de mala conducta ética. La JDR es miembro del Comité de Ética en Publicaciones (COPE) que provee una serie de lineamientos a las revistas sobre cómo resolver diferentes tópicos de mala conducta ética. Los investigadores pueden acceder a esta plataforma, que incluso tiene salas de chat y grupos de discusión. También seguimos las recomendaciones del Comité Internacional de Editores de Revistas Médicas. Así mismo, en las sesiones anuales de la $I A D R$ doy un Hands-on sobre tópicos selectos en mala conducta ética, esto está disponible en la página web de la $J D R$.

La mayoría de investigadores en el área de Odontología sueñan con que la JDR les acepte un artículo, pero ¿ustedes, los editores de las revistas, compiten para publicar investigaciones que les interesan?

Es una muy buena pregunta, nosotros los editores estamos en busca de nuevos hallazgos y nueva información que tenga un impacto global. Algunas veces las publicaciones involucran temas que pueden no ser tan novedosos. Sin embargo, son también publicables los estudios clínicos largos con resultados definitivamente contundentes. Tratamos de identificar estos trabajos. Podría decir que una de nuestras dificultades es tener que rechazar muy buenos artículos y de una alta calidad metodológica. Es probable que una de las principales razones de rechazo no sea por la calidad sino más bien porque el impacto del artículo es demasiado específico y compete a las diferentes especialidades como patología, endodoncia, periodoncia más no a la comunidad odontológica global. Todos los editores publican significativamente temas relacionados a sus líneas de investigación; en mi caso, como periodoncista, gran parte de mis publicaciones son enviadas a revistas de esta especialidad, cuando no alcanza un impacto global. Por otro lado, promovemos que todos los trabajos con un impacto más amplio se envíen a la $J D R$.
De todas las actividades programadas en este año por los 100 años de la JDR, ¿cuál es la que considera más significativa?

Esta es una muy buena pregunta, ya que pensamos que todas las actividades son muy importantes. Es por ello, que cada mes seleccionamos uno o dos artículos selectos. Por ejemplo, en el mes de julio publicamos un doble número sobre los mecanismos de las fluorizaciones, salud pública, genética y enfermedades orales, y biomateriales. También hemos publicado sobre salud sistémica oral y cómo esta área se ha transformado en los últimos 100 años. Genética y Bioingeniería son áreas que están en crecimiento que han sido inmersas en el proyecto del genoma humano y aún están en desarrollo versus investigaciones sobre salud sistémica o los trabajos realizados en flúor, con hallazgos fundamentales en los últimos cincuenta ańos y siguen en desarrollo. En el caso de la Genética, tenemos estudios sobre precisión diagnóstica y que están tomando cada vez más importancia. Dentro de poco, también publicaremos calidad de vida en odontología, neurofisiología; es divertido ver todos estos artículos publicarse durante este año. También me gustaría comentar acerca de la publicación de los " $J D R$ historical highlights". En cada número, Nicholas Jakubovics, quien es uno de nuestros editores asociados, se esfuerza en resaltar los puntos a recordar sobre trabajos realizados hace 100 años y cómo reflexionar acerca de las diferentes metodologías que hemos construido a través de los años y su impacto en nuestros campos de acción.

¿Cómo formar un editor de revista cientifica? ¿Qué recomendaciones daría a los jóvenes editores que recién se inician en este trabajo?

Me gusta esa pregunta porque creo que el ser editor, revisor o autor en una revista, todas son facetas gratificantes de nuestra vida profesional. Desde mi experiencia, el proceso comienza por entender cómo lograr la aceptación de nuestros artículos y todo lo que comprende llegar a esta etapa final; cómo se reúnen las diferentes partes de un artículo, el desarrollarlo identificando los datos de la investigación, y el trabajar en la redacción final del artículo. En particular, yo no fui un escritor nato, me tomaron muchos ańos el poder desarrollar mis habilidades en redacción. Tuve grandes mentores que me motivaron, entendí también que los comentarios críticos que recibía luego de enviar una publicación a una revista me ayudaban a crecer. Y si hablamos de autores latinoamericanos, el inglés no es su lengua materna, el solo hecho de relacionarse con otros pares puede ser de mucha ayuda. Pero nuevamente, en primer lugar, creo que la base está en ser un buen autor y el tener esta experiencia enriquecedora. Tenemos que tratar de entender la decepción tras un envío no exitoso de un manuscrito. Cada año yo publico varios artículos, pero durante el año tengo algunos trabajos que son rechazados, es decepcionante, pero se aprende de cada uno de los rechazos. A veces, hay alguna falta de comprensión por parte de los revisores o del editor y lo que he aprendido de este proceso es continuar mejorando la comu- 
nicación de la información y cómo esta información es procesada por el editor y por los revisores. Entonces, el ser un buen autor y desarrollar las habilidades en redacción, tomando las críticas constructivamente, luego tomar la oportunidad de ser un revisor. La característica común de nuestros editores asociados es que todos son revisores sobresalientes que se toman el tiempo de revisar críticamente la calidad de los trabajos analizados. La $J D R$ ha sido muy afortunada en tener muchos editores asociados de muy alto nivel: Joy Richman, Dana Graves, Gustavo Garlet, Christopher H. Fox, es un equipo maravilloso. Todos empezaron como autores proactivos que adquirieron experiencia como revisores y crecieron para llegar a ser editores.

¿Qué opinión tiene sobre el factor de impacto que se utiliza para evaluar la calidad de las revistas cientificas?

Es una buena pregunta, el factor de impacto es una de varias medidas para identificar la importancia de un manuscrito. En mi opinión, se le está dando demasiado valor, hay muchas formas de poder determinar la importancia de una publicación. En primer lugar depende de la comunidad, depende también del alcance del trabajo. En cuanto al factor de impacto aceptado en los últimos 40 años, se enfatiza si un artículo ha sido citado en un periodo de dos años. La Odontología es relativamente un área específica, tenemos revistas con un factor de impacto por debajo de cinco. Una de las cosas que promuevo que los colegas hagan es no enfocarse solo en el factor de impacto, sino más bien en cómo el trabajo está ayudando metodológicamente en el área de estudio, cómo se va generando interés desde el campo de salud, cómo estar vigilantes en tópicos selectos. La $J D R$ ha implementado una forma de medir el impacto científico que se ha desarrollado en los últimos años y mide la influencia científica de un artículo científico. También incluye un dato numérico que corresponde a las citaciones, número de descargas completas, la promoción en medios, entre otros factores. Con los años creo que encontraremos más formas de evaluar el factor de impacto.

¿Podría dar algún consejo a los jóvenes investigadores de Latinoamérica?

Para la gente joven, me gustaría recomendarles que lean tanto como puedan, que trabajen con asesores de calidad, que creen alianzas con personas en las que se puedan apoyar y que los ayuden a crecer, que cuando sea necesario critiquen su trabajo y que los ayuden a

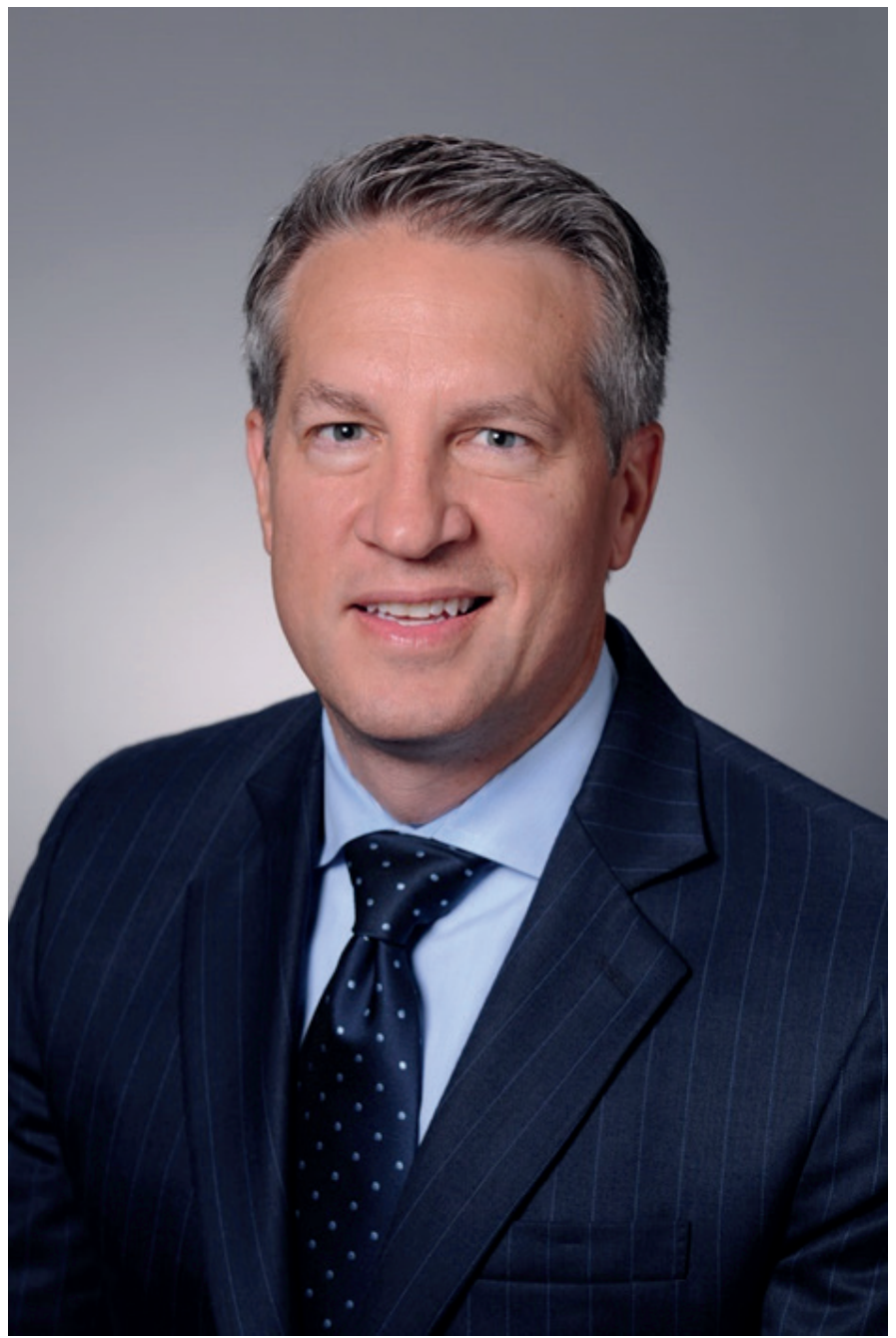

William Giannobile, editor jefe de la JDR, reflexiona sobre el sólido proceso de revisión por pares que permite que la JDR sea una fuente confiable de investigación para una amplia comunidad científica. 
decidir cuándo un artículo está listo para su publicación. No es importante publicar tantos artículos como sea posible sino más bien realizar investigaciones de alta calidad. Un buen mentor, los ayudará a formular sus investigaciones y a lograr una buena publicación teniendo la oportunidad de presentarlo en congresos científicos, compartiendo su información y aprendiendo de nuestros colegas. El leer y estar al día con las últimas publicaciones podría ayudar en el camino a ser un mejor investigador. Finalmente, es importante realizar intercambios interuniversitarios con universidades de habla inglesa. En la Universidad de Michigan tenemos alumnos de Brasil, Europa y de otras regiones como Chile, Argentina, Ecuador, México. Nuestra investigación no estaría en el nivel alcanzado sino fuera por todos nuestros colaboradores internacionales. El tener investigadores que inspiran y que comparten tiempo con nosotros, es de alguna manera parte de la internacionalización, filosofía que nos ayuda a trabajar en conjunto para avanzar en nuestras especialidades.

A futuro ¿cuáles son las tendencias de investigación en Odontologia?

Desde mi punto de vista esto va cambiando, es muy dinámico. En Odontología han existido muchos cambios en los últimos 3 a 5 años. Podemos mencionar muchos avances, en especial en el área de ciencias de los materiales, el acceso a grandes bases de datos y el poder analizar los datos con una alta calidad y precisión. En la actualidad se está trabajando en el área de la neurociencias y la genética con estudios innovadores. Finalmente, en áreas específicas como son microbiología, caries dental o enfermedad periodontal; hay diferentes oportunidades y tópicos de interés que son prometedores para los jóvenes que empiezan en este fascinante mundo de la investigación clínica. 
